\title{
Fourth Order Fitted Scheme for Second Order Singular Perturbation Boundary Value Problems
}

\author{
Awoke Andargie \\ Bahir dar University, Department of Mathematics, P.O.Box 79, Bahirdar, Ethiopia
}

\begin{abstract}
Fitted fourth order central difference scheme is presented for solving singularly perturbed two-point boundary value problems with the boundary layer at one end point. A fitting factor is introduced in a tri-diagonal finite difference scheme and is obtained from the theory of singular perturbations. Thomas Algorithm is used to solve the system and its stability is investigated. To demonstrate the applicability of the method, we have solved linear and nonlinear problems. From the results, it is observed that the present method approximates the exact solution very well.
\end{abstract}

Keywords Singular Perturbation Problems, Finite Differences, Fitted Scheme

\section{Introduction}

There are varieties of physical processes in which a boundary layer may arise in the solutions for certain parameter ranges. These types of problems may be generally characterized as singular perturbation problems, and the parameter is termed as the perturbation parameter. Detailed theory and analytical discussion on singular perturbation problems, can be referred in Bender and Orsazag[1], Kevorkian and Cole[3], Nayfeh[5-6], O'Mally[7] and Van Dyke[12] and for exponential fitted methods Miller,J.J.H., O'Riordan,E. and Shishkin, G.I.[4], Y.N.Reddy, P.Pramod. Chakravarthy[9] and Reddy Y.N., Awoke A.[10-11].

Fitted stable fourth order scheme is presented for solving singularly perturbed two-point boundary value problems with the boundary layer at one end point. A fitting factor is introduced in a tri-diagonal finite difference scheme and is obtained from the theory of singular perturbations. Thomas Algorithm is used to solve the system and its stability is investigated. To demonstrate the applicability of the method, we have solved several linear and nonlinear problems. From the results, it is observed that the present method approximates the exact solution very well.

\section{Fourth Order Finite Difference Method}

Consider a linear singularly perturbed two-point boundary value problem of the form:

* Corresponding author:

awoke248@yahoo.com (Awoke Andargie)

Published online at http://journal.sapub.org/ajcam

Copyright $(\underset{2}{2012}$ Scientific \& Academic Publishing. All Rights Reserved

$$
\begin{aligned}
& \varepsilon y^{\prime \prime}(x)+a(x) y^{\prime}(x)+b(x) y(x)=f(x), \mathrm{x} \in[0,1] \\
& \text { with } \mathrm{y}(0)=\alpha \\
& \text { and } \mathrm{y}(1)=\beta
\end{aligned}
$$

where $\varepsilon$ is a small positive parameter $(0<\varepsilon<<1)$ and $\alpha, \beta$ are known constants. We assume that $\mathrm{a}(\mathrm{x}), \mathrm{b}(\mathrm{x})$ and $\mathrm{f}(\mathrm{x})$ are sufficiently continuously differentiable functions in $[0,1]$. Further more, we assume that $b(x) \leq 0, a(x) \geq M>0$ throughout the interval[$[0,1]$, where $\mathrm{M}$ is some positive constant.

A finite difference scheme is often a convenient choice for the numerical solution of two point boundary value problems, because of their simplicity. Let us divide the interval[0,1] in to $\mathrm{N}$ equal parts, each of length $\mathrm{h}$ where $h=1 / N$ and then we have $x_{i}=i h, \mathrm{i}=0,1, \ldots, \mathrm{N}$. For simplicity, let us denote $\begin{aligned} & a\left(x_{i}\right)=a_{i}, b\left(x_{i}\right)=b_{i}, f\left(x_{i}\right)=f_{i}, y\left(x_{i}\right) \\ & =y_{i}, y^{\prime}\left(x_{i}\right)=y_{i}^{\prime} \text { and } \mathrm{y}^{\prime \prime}\left(\mathrm{x}_{\mathrm{i}}\right)=y_{i}^{\prime \prime} .\end{aligned}$

Assuming that $y$ has continuous fourth order derivatives in the interval $[0,1]$, by using Taylor series expansion we obtain the central difference formulas for $y_{i}^{\prime}, y_{i}^{\prime \prime}$ given by.

$$
y_{i}^{\prime} \cong \frac{y_{i+1}-y_{i-1}}{2 h}-\frac{h^{2}}{6} y_{i}^{\prime \prime \prime}+R_{1}
$$

and

$$
y_{i}^{\prime \prime} \cong \frac{y_{i+1}-2 y_{i}+y_{i-1}}{h^{2}}-\frac{h^{2}}{12} y_{i}^{(4)}+R_{2}
$$

where, $R_{1}=-\frac{h^{4} y^{(5)}(\eta)}{5 !}$ and $R_{2}=-\frac{2 h^{4} y^{(6)}(\xi)}{6 !}$ for $\xi, \eta \in\left[x_{i-1}, x_{i+1}\right]$.

From (1), we have

$$
\varepsilon y^{\prime \prime}=f(x)-a(x) y^{\prime}-b(x) y
$$

Differentiating both sides of equation (5) with respect to $\mathrm{x}$, we get

$$
y^{\prime \prime \prime}=\frac{1}{\varepsilon}\left(f^{\prime}-a y^{\prime \prime}-\left(a^{\prime}+b\right) y^{\prime}-b^{\prime} y\right)
$$

Differentiating twice both sides of equation (5) with respect to $\mathrm{x}$, we get 


$$
y^{(4)}=\frac{1}{\varepsilon}\left(f^{\prime \prime}-a y^{\prime \prime \prime}-\left(2 a^{\prime}+b\right) y^{\prime \prime}-\left(a^{\prime \prime}+2 b^{\prime}\right) y^{\prime}-b^{\prime \prime} y\right)
$$

By substituting $y_{i}^{\prime}, y_{i}^{\prime \prime}$ from equations (3) and (4) in (1) at $x=x_{i}$, we get the central difference approximation in a form that includes all the $o\left(h^{2}\right)$ error terms:

$$
\varepsilon\left(\frac{y_{i+1}-2 y_{i}+y_{i-1}}{h^{2}}-\frac{h^{2}}{12} y_{i}^{(4)}\right)+a_{i}\left(\frac{y_{i+1}-y_{i-1}}{2 h}-\frac{h^{2}}{6} y_{i}^{\prime \prime \prime}\right)+b_{i} y_{i}=f_{i}
$$

where, $y_{i}^{\prime \prime \prime}$ and $y_{i}^{(4)}$ are defined accordingly in equations (6) and (7) respectively. Using equations (3, 4, 6 \& 7) in equation (8), we get the fourth order scheme

$$
\begin{aligned}
& {\left[\frac{\varepsilon}{h^{2}}+\frac{a_{i}^{2}}{6 \varepsilon}-\frac{\left(2 a_{i}^{\prime}+b_{i}\right)}{12}\right]\left(y_{i+1}-2 y_{i}+y_{i-1}\right)} \\
& +\left[\frac{h a_{i}\left(a_{i}^{\prime}+b_{i}\right)}{12 \varepsilon}+\frac{h\left(a_{i}^{\prime \prime}+2 b_{i}^{\prime}\right)}{24}+\frac{a_{i}}{2 h}\right]\left(y_{i+1}-y_{i-1}\right) \\
& +\left[\frac{h^{2} a_{i} b_{i}^{\prime}}{6 \varepsilon}+\frac{h^{2} b_{i}^{\prime \prime}}{12}+b_{i}\right] y_{i}+R=f_{i}+\frac{h^{2} f_{i}^{\prime \prime}}{12}+\frac{h^{2} a_{i} f_{i}^{\prime}}{6 \varepsilon}, \quad \mathrm{i}=1,2, . ., \mathrm{N}-1
\end{aligned}
$$

Where, $R=R_{1}+R_{2}=O\left(h^{4}\right)$, from equation (4). For detail discussions of the method refer Joshua Y.Choo and David H.Schultz[2].

\section{Fitted Fourth Order Scheme}

A difference scheme with a fitting factor containing exponential functions is known as exponentially fitted difference scheme. From the theory of singular perturbations it is known that the solution of (1)-(2) is of the form [cf. O' Malley[7]; pp 22-26]

$$
y(x)=y_{0}(x)+\frac{a(0)}{a(x)}\left(\alpha-y_{0}(0)\right) e^{-\int_{0}^{x}\left(\frac{a(x)}{\varepsilon}-\frac{b(x)}{a(x)}\right) d x}+O(\varepsilon)
$$

where $y_{0}(x)$ is the solution of

$$
a(x) y_{0}^{\prime}(x)+b(x) y_{0}(x)=f(x), \quad y_{0}(1)=\beta
$$

By taking first terms of the Taylor's series expansion for $\mathrm{a}(\mathrm{x})$ and $\mathrm{b}(\mathrm{x})$ about the point ' 0 ', (10) becomes,

$$
y(x)=y_{0}(x)+\left(\alpha-y_{0}(0)\right) e^{-\left(\frac{a(0)}{\varepsilon}-\frac{b(0)}{a(0)}\right) x}+O(\varepsilon)
$$

Now we divide the interval $[0,1]$ into $\mathrm{N}$ equal parts with constant mesh length $\mathrm{h}$. Let $0=\mathrm{x}_{0}, \mathrm{x}_{1}, \mathrm{x}_{2}, \ldots \mathrm{x}_{\mathrm{N}}=1$ be the mesh points. Then we have $x_{i}=i h ; i=0,1,2, \ldots, N$.

From (11) we have

$$
y(i h)=y_{0}(i h)+\left(\alpha-y_{0}(0)\right) e^{-\left(\frac{a(0)}{\varepsilon}-\frac{b(0)}{a(0)}\right) i h}+O(\varepsilon) \text {. }
$$

Therefore

$$
\begin{aligned}
& \lim _{h \rightarrow 0} y(i h)=y_{0}(0)+\left(\alpha-y_{0}(0)\right) e^{-\left(\frac{a^{2}(0)-\varepsilon b(0)}{a(0)}\right) i \rho}, \\
& \text { where } \rho=\frac{h}{\varepsilon}
\end{aligned}
$$

Now, we consider the stable fourth order central difference scheme (8) and introduce the fitting factor $\sigma(\rho)$ :

$$
\begin{aligned}
& \sigma(\rho)\left[\frac{\varepsilon}{h^{2}}+\frac{a_{i}^{2}}{6 \varepsilon}-\frac{\left(2 a_{i}^{\prime}+b_{i}\right)}{12}\right]\left(y_{i+1}-2 y_{i}+y_{i-1}\right) \\
& +\left[\frac{h a_{i}\left(a_{i}^{\prime}+b_{i}\right)}{12 \varepsilon}+\frac{h\left(a_{i}^{\prime \prime}+2 b_{i}^{\prime}\right)}{24}+\frac{a_{i}}{2 h}\right]\left(y_{i+1}-y_{i-1}\right)
\end{aligned}
$$

$+\left[\frac{h^{2} a_{i} b_{i}^{\prime}}{6 \varepsilon}+\frac{h^{2} b_{i}^{\prime \prime}}{12}+b_{i}\right] y_{i}=f_{i}+\frac{h^{2} f_{i}^{\prime \prime}}{12}+\frac{h^{2} a_{i} f_{i}^{\prime}}{6 \varepsilon}, \quad \mathrm{i}=1,2, . ., \mathrm{N}-1$

Rewriting (13), we have

$$
\begin{gathered}
{\left[\frac{\sigma \varepsilon}{h^{2}}+\frac{a_{i}^{2}}{6 \varepsilon}-\frac{\sigma\left(2 a_{i}^{\prime}+b_{i}\right)}{12}\right]\left(y_{i+1}-2 y_{i}+y_{i-1}\right)} \\
+\left[\frac{h a_{i}\left(a_{i}^{\prime}+b_{i}\right)}{12 \varepsilon}+\frac{h\left(a_{i}^{\prime \prime}+2 b_{i}^{\prime}\right)}{24}+\frac{a_{i}}{2 h}\right]\left(y_{i+1}-y_{i-1}\right) \\
+\left[\frac{h^{2} a_{i} b_{i}^{\prime}}{6 \varepsilon}+\frac{h^{2} b_{i}^{\prime \prime}}{12}+b_{i}\right] y_{i}=f_{i}+\frac{h^{2} f_{i}^{\prime \prime}}{12}+\frac{h^{2} a_{i} f_{i}^{\prime}}{6 \varepsilon}, \quad \mathrm{i}=1,2, . ., \mathrm{N}-1
\end{gathered}
$$

$y_{0}=\alpha ; y_{N}=\beta$; where $\sigma(\rho)$ is a fitting factor which is to be determined in such a way that the solution of (14) converges uniformly to the solution of (1)-(2).

Multiplying (14) by $\mathrm{h}$ and taking the limit as $\mathrm{h} \rightarrow 0$; we get

$$
\lim _{h \rightarrow 0}\left[\begin{array}{l}
\left(\frac{\sigma}{\rho}+\frac{\sigma a(0)^{2} \rho}{6}\right)(y((i+1) h)-2 y(i h)+y((i-1) h)) \\
+\frac{a(0)}{2}(y((i+1) h)-y((i-1) h))
\end{array}\right]=0
$$

By using (12) in (15) and simplifying, we get

$$
\lim _{h \rightarrow 0} S \sigma=\frac{a(0)}{2} \operatorname{coth}\left(\left(\frac{\mathrm{a}^{2}(0)-\varepsilon b(0)}{a(0)}\right) \frac{\rho}{2}\right)
$$

Where $S=\frac{6+a^{2}(0) \rho^{2}}{6 \rho}$

We have; $\sigma=\frac{a(0)}{2 S} \operatorname{coth}\left(\left(\frac{\mathrm{a}^{2}(0)-\varepsilon b(0)}{a(0)}\right) \frac{\rho}{2}\right)$

$\sigma$ given by (18) is the constant fitting factor.

From (14) we have

$$
\begin{gathered}
{\left[\frac{\sigma \varepsilon}{h^{2}}+\frac{\sigma a_{i}^{2}}{6 \varepsilon}-\frac{\sigma\left(2 a_{i}^{\prime}+b_{i}\right)}{12}-\frac{h a_{i}\left(a_{i}^{\prime}+b_{i}\right)}{12 \varepsilon}-\frac{h\left(a_{i}^{\prime \prime}+2 b_{i}^{\prime}\right)}{24}-\frac{a_{i}}{2 h}\right] y_{i-1}} \\
-\left[2\left[\frac{\sigma \varepsilon}{h^{2}}+\frac{\sigma a_{i}^{2}}{6 \varepsilon}-\frac{\sigma\left(2 a_{i}^{\prime}+b_{i}\right)}{12}\right]-\frac{h^{2} a_{i} b_{i}^{\prime}}{6 \varepsilon}-\frac{h^{2} b_{i}^{\prime \prime}}{12}-b_{i}\right] y_{i} \\
+\left[\frac{\sigma \varepsilon}{h^{2}}+\frac{\sigma a_{i}^{2}}{6 \varepsilon}-\frac{\sigma\left(2 a_{i}^{\prime}+b_{i}\right)}{12}-\frac{h a_{i}\left(a_{i}^{\prime}+b_{i}\right)}{12 \varepsilon}-\frac{h\left(a_{i}^{\prime \prime}+2 b_{i}^{\prime}\right)}{24}-\frac{a_{i}}{2 h}\right] y_{i+1} \\
=f_{i}+\frac{h^{2} f_{i}^{\prime \prime}}{12}+\frac{h^{2} a_{i} f_{i}^{\prime}}{6 \varepsilon}, \mathrm{i}=1,2, . ., \mathrm{N}-1
\end{gathered}
$$

where the fitting factor $\sigma$ is given by (18).

The equivalent three term recurrence relation of equation (19) is given by:

$$
E_{i} y_{i-1}-F_{i} y_{i}+G_{i} y_{i+1}=H_{i} ; \mathrm{i}=1,2,3,
$$

where

$$
\begin{gathered}
E_{i}=\frac{\sigma \varepsilon}{h^{2}}+\frac{\sigma a_{i}^{2}}{6 \varepsilon}-\frac{\sigma\left(2 a_{i}^{\prime}+b_{i}\right)}{12}+\frac{h a_{i}\left(a_{i}^{\prime}+b_{i}\right)}{12 \varepsilon}+\frac{h\left(a_{i}^{\prime \prime}+2 b_{i}^{\prime}\right)}{24}+\frac{a_{i}}{2 h} \\
F_{i}=2\left[\frac{\sigma \varepsilon}{h^{2}}+\frac{\sigma a_{i}^{2}}{6 \varepsilon}-\frac{\sigma\left(2 a_{i}^{\prime}+b_{i}\right)}{12}\right]-\frac{h^{2} a_{i} b_{i}^{\prime}}{6 \varepsilon}-\frac{h^{2} b_{i}^{\prime \prime}}{12}-b_{i} \\
G_{i}=\frac{\sigma \varepsilon}{h^{2}}+\frac{\sigma a_{i}^{2}}{6 \varepsilon}-\frac{\sigma\left(2 a_{i}^{\prime}+b_{i}\right)}{12}-\frac{h a_{i}\left(a_{i}^{\prime}+b_{i}\right)}{12}-\frac{h\left(a_{i}^{\prime \prime}+2 b_{i}^{\prime}\right)}{24}-\frac{a_{i}}{2 h} \\
H_{i}=f_{i}+\frac{h^{2} f_{i}^{\prime \prime}}{12}+\frac{h^{2} a_{i} f_{i}^{\prime}}{6 \varepsilon}
\end{gathered}
$$

This gives us the tri-diagonal system which can be solved easily by Thomas Algorithm.

\section{Thomas Algorithm}

A brief discussion on solving the tri-diagonal system using Thomas algorithm is presented as follows:

Consider the scheme given in (20): 


$$
E_{i} y_{i-1}-F_{i} y_{i}+G_{i} y_{i+1}=H_{i} ; \mathrm{i}=1,2,3,
$$

subject to the boundary conditions

$$
\begin{aligned}
& y_{0}=y(0)=\alpha \\
& y_{N}=y(1)=\beta
\end{aligned}
$$

We set $y_{i}=W_{i} y_{i+1}+T_{i}$ for $\mathrm{i}=\mathrm{N}-1, \mathrm{~N}-2, \ldots .2,1$.

where $W_{i}=W\left(x_{i}\right)$ and $T_{i}=T\left(x_{i}\right)$ which are to be determined. From (22), we have

$$
y_{i-1}=W_{i-1} y_{i}+T_{i-1}
$$

By substituting (23) in (20), we get

$$
\begin{aligned}
& E_{i}\left(W_{i-1} y_{i}+T_{i-1}\right)-F_{i} y_{i}+G_{i} y_{i+1}=H_{i} . \\
& y_{i}=\left(\frac{G_{i}}{F_{i}-E_{i} W_{i-1}}\right) y_{i+1}+\left(\frac{E_{i} T_{i-1}-H_{i}}{F_{i}-E_{i} W_{i-1}}\right)
\end{aligned}
$$
tions

By comparing (24) and (22), we get the recurrence rela-

$$
\begin{gathered}
W_{i}=\left(\frac{G_{i}}{F_{i}-E_{i} W_{i-1}}\right) \\
T_{i}=\left(\frac{E_{i} T_{i-1}-H_{i}}{F_{i}-E_{i} W_{i-1}}\right)
\end{gathered}
$$

To solve these recurrence relations for $\mathrm{i}=0,1,2,3,, \mathrm{~N}-1$, we need the initial conditions for $W_{0}$ and $T_{0}$. For this we take $y_{0}=\alpha=W_{0} y_{1}+T_{0}$. We choose $W_{0}=0$ so that the value of $T_{0}=\alpha$.With these initial values, we compute $W_{i}$ and $T_{i}$ for $\mathrm{i}=1,2,3, \ldots, \mathrm{N}-1$ from $(25)$ in forward process, and then obtain $y_{i}$ in the backward process from (22)and (21b).

\section{Stability Analysis}

We will now show that the algorithm is computationally stable. By stability, we mean that the effect of an error made in one stage of the calculation is not propagated into larger errors at later stages of the calculations. Let us now examine the recurrence relation given by (25a). Suppose that a small error $e_{i-1}$ has been made in the calculation of $W_{i-1}$; then, we have

$$
\begin{gathered}
\bar{W}_{i-1}=W_{i-1}+e_{i-1} \text { and we are actually calculating } \\
\bar{W}_{i}=\left(\frac{G_{i}}{F_{i}-E_{i} \bar{W}_{i-1}}\right)
\end{gathered}
$$

From (26) and (25a), we have

$$
\begin{aligned}
& e_{i}=\left(\frac{G_{i}}{F_{i}-E_{i}\left(W_{i-1}+e_{i-1}\right)}\right)-\left(\frac{G_{i}}{F_{i}-E_{i} W_{i-1}}\right) \\
& =\left(\frac{G_{i} E_{i} e_{i-1}}{\left(F_{i}-E_{i}\left(W_{i-1}+e_{i-1}\right)\right)\left(F_{i}-E_{i} W_{i-1}\right)}\right) \\
& =\left(\frac{W_{i}^{2} E_{i}}{G_{i}}\right) e_{i-1}
\end{aligned}
$$

under the assumption that the error is small initially. From the assumptions made earlier that $\mathrm{a}(\mathrm{x})>0, \mathrm{~b}(\mathrm{x}) \leq 0$ and its derivatives also non-positive, we have

$$
F_{i} \geq E_{i}+G_{i} ; \mathrm{i}=1,2,3, \ldots, \mathrm{N}-1
$$

Form (25a) we have $W_{1}=\frac{G_{1}}{F_{1}}<1$, since $\mathrm{F}_{1}>\mathrm{G}_{1}$

$$
\begin{gathered}
W_{2}=\frac{G_{2}}{F_{2}-E_{2} W_{1}}<\frac{W_{1} G_{2}}{F_{2}-E_{2}} ; \text { since } \mathrm{W}_{1}<1, \\
<\frac{G_{2}}{E_{2}+G_{2}-E_{2}}=1 ; \text { since } \mathrm{F}_{2} \geq \mathrm{E}_{2}+\mathrm{G}_{2}
\end{gathered}
$$

Successively, it follows that $\left|e_{i}\right|=\left|W_{i}\right|^{2}\left|\frac{E_{i}}{G_{i}}\right|\left|e_{i-1}\right|<$ $\left|e_{i-1}\right|$, since $\left|E_{i}\right| \leq\left|G_{i}\right|$.

Therefore the recurrence relation (25a) is stable. Similarly we can prove that the recurrence relation (25b) is also stable. Finally the convergence of the Thomas Algorithm is ensured by the condition $\left|W_{i}\right|<1, \mathrm{i}=1,2,3, \ldots, \mathrm{N}-1$.

\section{Numerical Examples}

\subsection{Example}

Consider the following homogeneous singular perturbation problem from Bender and Orszag[[1], page 480; problem 9.17 with $\alpha=0$ ]

$$
\varepsilon y^{\prime \prime}(x)+y^{\prime}(x)-y(x)=0 \quad ; \quad \mathrm{x} \in[0,1] \text {, with } \quad \mathrm{y}(0)=1 \quad \text { and }
$$
$\mathrm{y}(1)=1$.

The exact solution is given by:

$$
y(x)=\frac{\left[\left(e^{m_{2}}-1\right) e^{m_{1} x}+\left(1-e^{m_{1}}\right) e^{m_{2} x}\right]}{\left[e^{m_{2}}-e^{m_{1}}\right]}
$$

Where $\mathrm{m}_{1}=(-1+\sqrt{1+4 \varepsilon}) /(2 \varepsilon)$ and $\mathrm{m}_{2}=$ $(-1-\sqrt{1+4 \varepsilon}) /(2 \varepsilon)$

Error plot for Example 4.1, $\varepsilon=10^{-3}, \mathrm{~h}=10^{-2}$

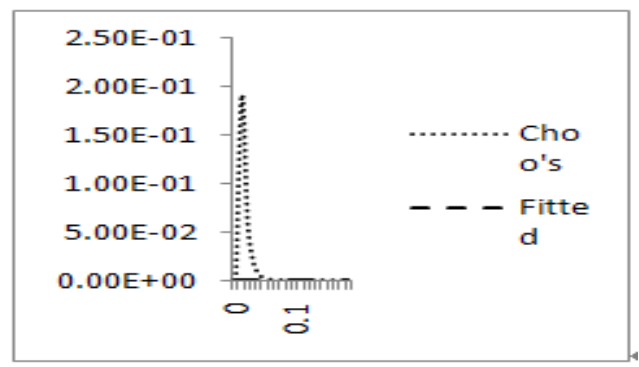

Error plot for example 4.1, $\varepsilon=10^{-4}, \mathrm{~h}=10^{-2}$.

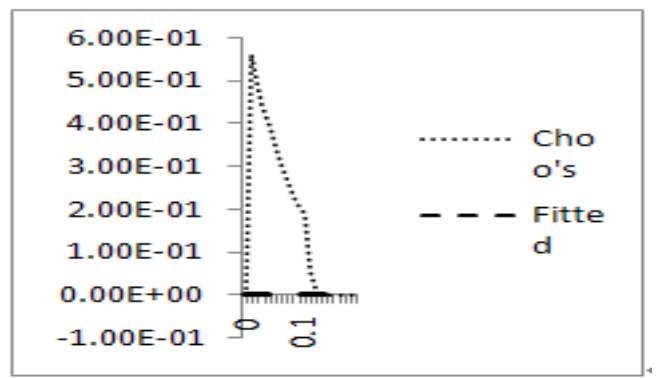

\subsection{Example}

Let us consider the following non-homogeneous singular perturbation problem from fluid dynamics for fluid of small viscosity, Reinhardt[[8], example 2]

$\varepsilon y^{\prime \prime}(x)+y^{\prime}(x)=1+2 x ; \mathrm{x} \in[0,1]$, with $\mathrm{y}(0)=0$ and $\mathrm{y}(1)=1$.

The exact solution is given by

$$
\mathrm{y}(\mathrm{x})=\mathrm{x}(\mathrm{x}+1-2 \varepsilon)+\frac{(2 \varepsilon-1)\left(1-e^{-x / \varepsilon}\right)}{\left(1-e^{-1 / \varepsilon}\right)}
$$


Error plot for Example 4.2, $\varepsilon=10^{-3}, \mathrm{~h}=10^{-2}$

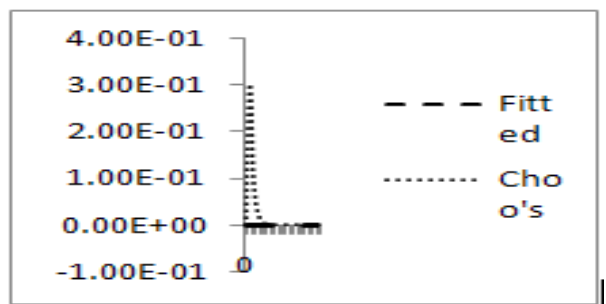

Errorplot for Example 4.2, $\varepsilon=10^{-4}, \mathrm{~h}=10^{-2}$

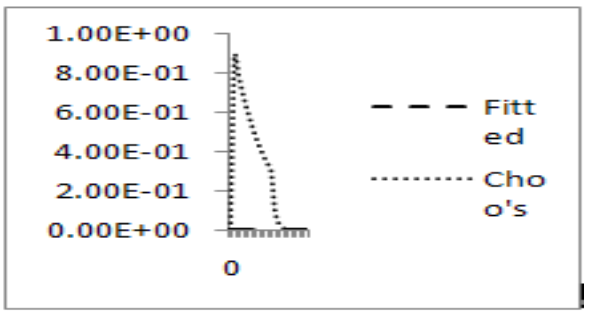

\section{Non-linear Problems}

Nonlinear singular perturbation problems were converted as a sequence of linear singular perturbation problems by using quasilinearization (Replacing the non-linear problem by a sequence of linear problems) method. The outer solution (the solution of the given problem by putting $\varepsilon=0$ ) is taken to be the initial approximation.

\subsection{Example}

Consider the following singular perturbation problem from Bender and Orszag[[1], page 463; equations: 9.7.1]

with $\mathrm{y}(0)=0$ and $\mathrm{y}(1)=0$.

$$
\varepsilon y^{\prime \prime}(x)+2 y^{\prime}(x)+e^{y(x)}=0 ; \mathrm{x} \in[0,1]
$$

The linear problem concerned to this example is

$$
\varepsilon y^{\prime \prime}(x)+2 y^{\prime}(x)+\frac{2}{x+1} y(x)=\left(\frac{2}{x+1}\right)\left[\log _{e}\left(\frac{2}{x+1}\right)-1\right]
$$

We have chosen to use Bender and Orszag's uniformly valid approximation[[1], page 463; equation: 9.7.6] for comparison, $y(x)=\log _{e}\left(\frac{2}{x+1}\right)-\left(\log _{e} 2\right) e^{-2 x / \varepsilon}$

For this example, we have boundary layer of thickness $\mathrm{O}(\varepsilon)$ at $\mathrm{x}=0$. [cf. Bender and Orszag[1]].

Error plot for Example 5.1, $\varepsilon=10^{-3}, \mathrm{~h}=10^{-2}$

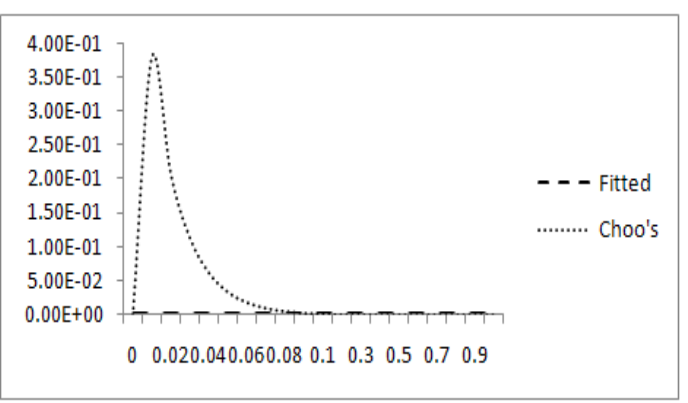

Error plot for Example 5.1, $\varepsilon=10^{-4}, \mathrm{~h}=10^{-2}$

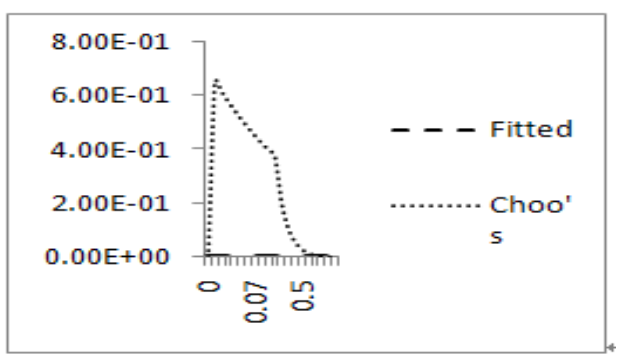

\subsection{Example}

Let us consider the following singular perturbation problem from Kevorkian and Cole[[3], page 56; equation 2.5.1] $\varepsilon y^{\prime \prime}(\mathrm{x})+\mathrm{y}(\mathrm{x}) \mathrm{y}^{\prime}(\mathrm{x})-\mathrm{y}(\mathrm{x})=0 ; \mathrm{x} \in[0,1]$, with $\mathrm{y}(0)=-1$ and $\mathrm{y}(1)=3.9995$

The linear problem concerned to this example is

$$
\varepsilon y^{\prime \prime}(x)+(x+2.9995) y^{\prime}(x)=x+2.9995
$$

We have chosen to use the Kevorkian and Cole's uniformly valid approximation[[3], pages 57 and 58; equations (2.5.5), (2.5.11) and (2.5.14)] for comparison,

$$
\mathrm{y}(\mathrm{x})=\mathrm{x}+\mathrm{c}_{1} \tanh \left(\left(\frac{\mathrm{c}_{1}}{2}\right)\left(\frac{\mathrm{x}}{\varepsilon}+\mathrm{c}_{2}\right)\right)
$$

Where $c_{1}=2.9995$ and $c_{2}=\left(1 / c_{1}\right) \log _{e}\left[\left(c_{1}-1\right) /\left(c_{1}+1\right)\right]$

For this example also we have a boundary layer of width $\mathrm{O}(\varepsilon)$ at $\mathrm{x}=0$ [cf. Kevorkian and Cole[3], pages 56-66].

The numerical results are given in table $4(\mathrm{a}), 4(\mathrm{~b})$ for $\varepsilon=10^{-3}$ and $10^{-4}$ respectively.

Error plot for Example 5.2, $\varepsilon=10^{-3}, \mathrm{~h}=10^{-2}$

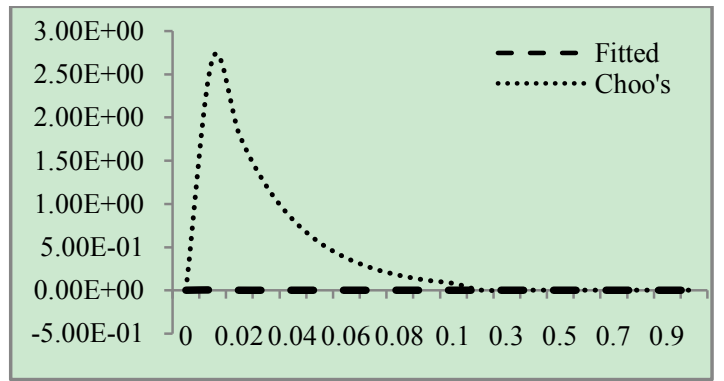

Error plot for Example 5.2, $\varepsilon=10^{-4}, \mathrm{~h}=10^{-2}$

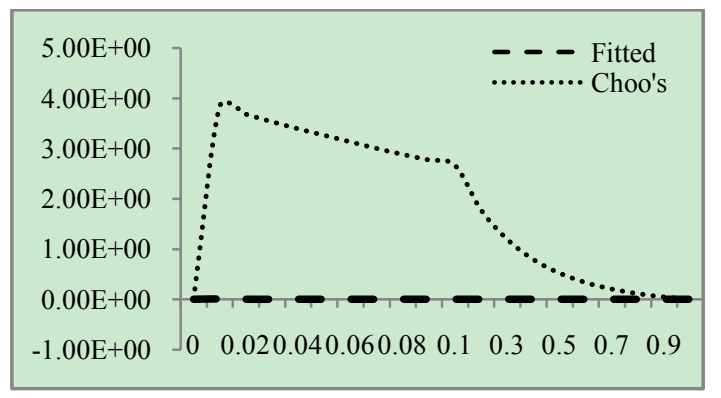

\section{Conclusions}

We have presented fitted fourth order finite difference 
method for solving singularly perturbed two-point boundary value problems The present fitted fourth order finite difference method for solving singularly perturbed two-point boundary value problems produce better approximation to the exact solution, specifically in the boundary layer region with step size $h>\varepsilon$, the perturbation parameter.

\section{REFERENCES}

[1] Bender, C.M. and Orsazag, S.A.(1978): Advanced Mathematical Methods for Scientists and Engineers, McGraw-Hill, New York

[2] Joshua Y.Choo and David H.Schultz(1993): Stable higher order methods for differential equations with small coefficients for the second order term, Computers Math. Applics: Vol.25,No.1,pp.105-123

[3] Kevorkian, J. and Cole, J.D. (1981): Perturbation Methods in Applied Mathematics, Springer-Verlag, New York

[4] Miller,J.J.H., O’Riordan,E. and Shishkin, G.I. (1996): Fitted Numerical Methods for Singular Perturbation Problems, World Scientific, River Edge, NJ
[5] Nayfeh A.H. (1981): Introduction to Perturbation Techniques, Wiley, New York

[6] Nayfeh A.H.: Perturbation Methods, Wiley, New York, 1979

[7] O'Malley, R.E. (1974): Introduction to Singular Perturbations, Academic Press, New York

[8] [8] Reinhardt, H.J. (1980): Singular Perturbations of difference methods for linear ordinary differential equations, Applicable Anal., 10, 53-70

[9] Reddy Y.N., Chakravarthy P.P. (2004): An exponentially fitted finite difference method for singular perturbation problems, Applied Mathematics and Computation 154, 83-101

[10] Reddy Y.N., Awoke A. (2007): An Exponentially Fitted Special second order finite difference method for singular perturbation problems, Applied Mathematics and Computation, 190, 1767-1782

[11] Reddy Y.N., Awoke A. (2007): Fitted fourth order tridiagonal finite difference method for singular perturbation problems, Applied Mathematics and Computation, 192, 90-100

[12] Van Dyk.M (1964): Perturbation Methods in Fluid Mechanics. Academic Press, New Y (1964): Perturbation Methods in Fluid Mechanics. Academic Press, New York 